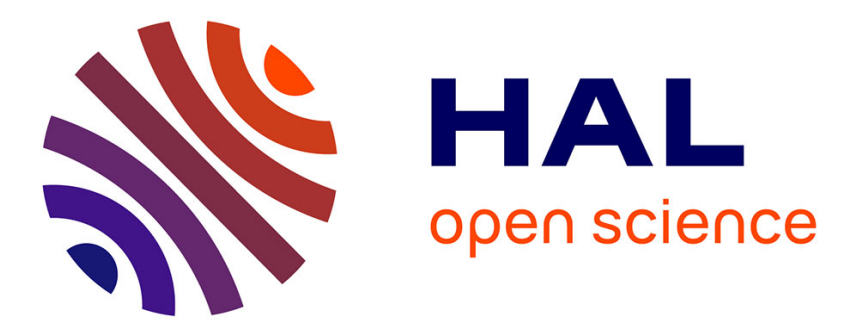

\title{
Mes Loisirs à l'heure des humanités numériques. Un journal d'événement au miroir des études sur les ego-documents
}

Pierre-Yves Beaurepaire

\section{- To cite this version:}

Pierre-Yves Beaurepaire. Mes Loisirs à l'heure des humanités numériques. Un journal d'événement au miroir des études sur les ego-documents. Histoire, économie et société, 2018, Dossier sur le Journal d'Hardy, 2018 (2), pp.8-17. hal-01819224

\section{HAL Id: hal-01819224 \\ https://hal.science/hal-01819224}

Submitted on 10 Jul 2018

HAL is a multi-disciplinary open access archive for the deposit and dissemination of scientific research documents, whether they are published or not. The documents may come from teaching and research institutions in France or abroad, or from public or private research centers.
L'archive ouverte pluridisciplinaire HAL, est destinée au dépôt et à la diffusion de documents scientifiques de niveau recherche, publiés ou non, émanant des établissements d'enseignement et de recherche français ou étrangers, des laboratoires publics ou privés. 


\title{
Mes Loisirs à l'heure des humanités numériques.
}

\section{Un journal d'événement au miroir des études sur les ego-documents}

\author{
Pierre-Yves Beaurepaire \\ Université Côte d'Azur, CMMC
}

\section{Introduction}

Dans un article pionnier paru en 1999, Valérie Goutal-Arnal, qui a réuni les premiers matériaux d'une biographie du libraire parisien, considérait que « le journal de S.-P. Hardy n'a rien d'un journal intime. ${ }^{1}$ Mais, depuis les travaux stimulants entrepris une décennie plus tard par le groupe de recherche du Centre national de la recherche scientifique «Ecrits du For Privé »- en référence à une contribution de Madeleine Foisil à 1'Histoire de la vie privée dirigée par Philippe Ariès et Georges Duby en $1986^{2}$ - cette affirmation mérite sans doute d'être nuancée, car l'écriture de soi emprunte des voies et des supports multiples, ${ }^{3}$ parfois même insoupçonnées, si l'on songe aux lames du parquet du château de Picomtal dans le département français

\footnotetext{
1 Valérie Goutal-Arnal, «"Mes loisirs ou journal d'événements tels qu'ils parviennent à ma connoissance". Chronique (1753-1789) du libraire Siméon-Prosper Hardy », Revue d'histoire moderne et contemporaine 1999/3 ( $\left.\mathrm{n}^{\circ} 46-3\right)$, p. 457.

${ }^{2}$ Madeleine Foisil, «L'écriture du for privé », Philippe Ariès et Georges Duby (dir.), L'Histoire de la vie privée, Paris, 1986, III, p. 331-369. Parmi les publications consacrées aux écrits du for privé, on se reportera notamment à Les Ecrits du for privé ; objets matériels, objets édités, sous la direction de Michel Cassan, Jean-Pierre Bardet, François-Joseph Ruggiu, Limoges, Presses de l'université de Limoges, 2007. Il s'agit du GDR 2649 labellisé par le Centre national de la Recherche scientifique en 2003 et renouvelé en 2007 sous le titre : «Les écrits du for privé en France de la fin du Moyen Age à 1914 ». La base de données est accessible à partir de l'adresse : http://inv.ecritsduforprive.humanum.fr/search-form.html

${ }^{3}$ On se reportera notamment à l'ouvrage conclusif de ce programme : Jean-Pierre Bardet, FrancoisJoseph Ruggiu (dir.), Les Écrits du for privé en France. De la fin du Moyen Âge à 1914, Paris, éditions du CTHS, 2015, Orientations et méthodes n ${ }^{\circ}$ 29, ainsi qu'à l'article de François-Joseph Ruggiu, « Les discours annalistiques comme discours de soi », Sylvie Mouysset, Jean-Pierre Bardet et FrançoisJoseph Ruggiu (dir.), Car c'est moy que je peins. Ecritures de soi, individus et liens sociaux, Toulouse, Méridiennes, 2011, p. 261-277 et au bref texte d'Isabelle Galichon, «Récit de soi », Témoigner. Entre histoire et mémoire, 117, 2014, http://temoigner.revues.org/1212 mis en ligne le $1^{\mathrm{er}}$ juin 2015, consulté le 20 décembre 2017.
} 
des Hautes-Alpes que le menuisier Joachim Martin a recouvertes de son écriture à la fin du XIXe siècle. ${ }^{4}$ Je voudrais donc proposer ici de lire Mes Loisirs au miroir des travaux sur les ego-documents, ${ }^{5}$ et montrer qu'à l'instar d'autres journaux d'événements, longtemps exploités comme de simples réservoirs à citations ${ }^{6}$, Mes Loisirs mérite d'être considéré avec tout l'intérêt que les chercheurs portent aujourd'hui aux écritures sans qualité, ordinaires.

\section{Du magasin à citations aux ressources de l'écriture sans qualité}

Hardy l'indique lui-même, il tient un journal d'événemens tels qu'ils parviennent à ma connoissance, souvent introduits par : «il se répand dans le public que », ou encore, « on rapporte que ». Il ne s'agit pas d'une écriture de l'intime, mais pour l'auteur de se faire le chroniqueur du temps, le diariste des événements, informations, bruits parvenus jusqu'à lui. Il s'agit donc bien d'un exemple de journal d'événements, que Christian Jouhaud, Dinah Ribard et Nicolas Schapira caractérisent dans Histoire Littérature Témoignage. Écrire les malheurs du temps en ces termes :

\footnotetext{
${ }^{4}$ Jacques-Olivier Boudon, Le plancher de Joachim. L'histoire retrouvée d'un village français, Paris, Belin, 2017.

${ }^{5}$ Néologisme forgé dans les années 1950 par l'historien néerlandais Jacob Presser, pour qui un egodocument désigne les écrits dans lesquels un auteur évoque sa vie personnelle et ses sentiments, soit tous les textes où affleure un ego. Sur l'œuvre de Presser, on pourra se reporter à Winfried Schulze, «Ego-Dokumente : Annäherung an den Menschen in der Geschichte? Vorüberlegungen für die Tagung «Ego Dokumente»», Winfried Schulze (dir.), Ego-Dokumente: Annäherung an den Menschen in der Geschichte, Akademic Verlag, Berlin, 1996, p. 11-30 ; ainsi qu'à Rudolf Dekker, « Jacques Presser's heritage : Egodocuments in the study of history », Memoria y civilizaciòn, 2002, $\mathrm{n}^{\circ} 5$, p. 13-37. Parmi les recherches consacrées aux ego-documents, on pourra consulter Pierre-Yves Beaurepaire et Dominique Taurisson (dir.), Les ego-documents à l'heure de l'électronique. Nouvelles approches des espaces et des réseaux relationnels, Montpellier, Presses universitaires de Montpellier, 2003. La European Scientific Foundation (Strasbourg) a consacré aux ego-documents un programme intitulé : Ego-documents in an European Context. First-person writings in Europe from the end of the Middle Ages to the beginning of the twentieth century.

${ }^{6}$ Voir notamment l'usage qu'en font Roland Mortier dans Le Dix-huitième siècle français au quotidien. Textes tirés des Mémoires, des Journaux et des Correspondances de l'époque, Bruxelles, éditions Complexe, 2002 et Arnaud de Maurepas dans : «L'oeil, l'oreille et la plume : la sensibilité testimoniale dans le Journal de Barbier (1717-1762)», Histoire, économie et société, 1991, $10^{\mathrm{e}}$ année, n 4, p. 491503.
} 
Les 'journaux d'événements' tendent à être mobilisés comme des témoignages fiables, sinon tout à fait transparents, sur ce qui se passait dans le public, à l'occasion d'un crime individuel ou collectif, d'un procès, d'une exécution, d'une répression. Parce que leurs auteurs affirment (et semblent en effet) recueillir ce qu'ils voient, entendent ou entendent dire, ils sont lus pour ce qu'ils disent de la manière dont les Parisiens recevaient les spectacles de la violence et les nouvelles sur les crimes et les châtiments. [...] La conclusion - à l'interrogation sur la nature de leurs écrits - est toujours que cette nature n'est en aucun cas littéraire, et que ces lettrés n'étaient ni des auteurs ni des acteurs : on ne cherche chez eux qu'un regard. ${ }^{7}$

Partant, on peut rapprocher Mes loisirs d'autres écrits familiers des historiens du XVIII ${ }^{\mathrm{e}}$ siècle en général et de Paris en particulier, notamment de ceux des chroniqueurs et diaristes Jean Buvat et Mathieu Marais pour le premier XVIII ${ }^{\mathrm{e}}$ siècle, du Journal de Jean Buvat couvrant les années 1715-1723, soit exactement la Régence de Philippe duc d'Orléans, et de celui de Mathieu Marais prenant en quelque sorte le relais, de 1723 à 1737. Dans le premier, Buvat adopte la position classique - et donc attendue - de la modestie du scripteur qui ne saurait se considérer comme historien. Il insiste dans la «préface » sur le fait qu'il se contente d'enregistrer les événements, de collationner les informations, et qu'il s'est bien gardé de tout commentaire personnel :

Comme ce journal est à proprement parler une simple collection de faits et de pièces pour une histoire plus étendue et plus circonstanciée, on s'est dispensé de faire aucune réflexion sur les divers événements qui se sont vus pendant la Régence, afin de laisser aux personnes habiles la liberté d'en faire de la manière qu'elles jugeront à propos. On y a ramassé tout ce qu'on a pu ; quelques infirmités survenues, quoique de peu de durée, ont pu faire échapper quelques circonstances ; ce qu'on aura la bonté d'excuser. ${ }^{8}$

Bien évidemment, l'historien ne doit pas prendre cette convention au pied de la lettre, car les événements auxquels Buvat assiste font bien l'objet d'une mise en écriture, celle de la littérature de témoignage, qui laisse filtrer l'opinion de l'auteur, son interprétation, les rapprochements auxquels il procède, voire l'appropriation -

\footnotetext{
${ }^{7}$ Christian Jouhaud, Dinah Ribard et Nicolas Schapira, Histoire Littérature Témoignage. Écrire les malheurs du temps, Paris, Gallimard, Folio histoire, 2009, p. 315-316. A noter que les auteurs fondent leur critique en faisant expressément référence à Pascal Bastien et à Hardy.

${ }^{8}$ Jean Buvat, Journal de la Régence : 1715-1723, «préface », Paris, Plon, 1865, p. 35. Les quatre volumes autographes du Journal de Buvat sont conservés à la BnF, manuscrits français 10281-10284.
} 
réemploi de techniques d'écriture littéraire. C'est d'autant plus vrai pour Buvat - qui est fondamentalement un homme du livre... comme l'est Hardy. Buvat ne quitte en effet la Bibliothèque du roi qu'à sa mort, en 1729.

Or, le renouvellement des objets et des problématiques scientifiques qui privilégient les enjeux sociaux, culturels et publics de l'écriture des particuliers sans condition, nous donne l'occasion de «réinventer» ces sources, en proposant de nouveaux questionnaires et de nouvelles mises en perspective, associant ou non histoire et littérature. Comme l'écrivent Philippe Artières et Dominique Kalifa dans la présentation du dossier «L'historien et les archives personnelles : Pas à pas », paru en 2002 dans la revue Sociétés \& Représentations :

Depuis une trentaine d'années, et notamment à la suite de la publication par Michel Foucault des Mémoires du parricide Pierre Rivière, l'historiographie française a été marquée par une valorisation accrue des sources autobiographiques. Jacques-Louis Ménétra, Nicolas Contat, Charles Noiret, Caroline Brame, Jean-Baptiste Dumay, Louis Barthas, Jeanne Bouvier, et quelques autres individus jusque là sans visage ni épaisseur sont venus peupler l'histoire des derniers siècles, et la doter de ce que Foucault appelait le «marmonnement du monde ». L'attention progressivement portée à la question du "témoignage » et à ses relations au "vrai », les divers déplacements proposés ou opérés par la microstoria, la réévaluation du rôle et des compétences des acteurs, ou encore la part croissante consacrée à l'écriture dans la construction des objets historiques, ont largement contribué à accentuer ce mouvement. ${ }^{9}$

Il n'est bien sûr pas question de faire de Siméon-Prosper Hardy un nouveau Jacques-Louis Ménétra, qui, lui, rédige une autobiographie riche en notations personnelles. En effet, le projet d'écriture de ce dernier est bien celui d'une écriture du for privé populaire qui cherche ses marques, sa nature et se démarque des écritures des gens bien nés : «Journal de ma vie écrit pour moi en l'an 1764 / Ménétra / le tout sans ostentation et sans réflexion / Ecrire la vérité selon moi cela doit être ni parler d'armes

\footnotetext{
${ }^{9}$ Philippe Artières et Dominique Kalifa, « Présentation. L'historien et les archives personnelles : Pas à pas », Sociétés \& Représentations, 2002/1 - n 13, p. 7.
} 
et de / blasons oublier ce qu'étaient ses ancêtres et de ces vains titres n'en / décorer son nom ». Comme l'écrit Daniel Roche :

« Mémoires », « Souvenirs », «Confessions », « Journal de ma vie », «Histoire»? Des mots qui ponctuent les publications littéraires, «préface», «dédicace », «épître » et «profession de foi » surgissent tout naturellement mais s'il les connaît il n'en maîtrise pas totalement l'usage et son doute se traduit dans la sous-qualification qu'il revendique. Ses écrits sont des 《 griffonnages » un « fatras »; la tentation de la destruction l'obsède, tout cela est « bon à jeter $[\ldots]$ au feu $[\ldots]$ à renier $[\ldots]$ à effacer $[\ldots]$ à biffer $»^{10}$

Pour autant, une lecture attentive de Mes Loisirs montre que sur des thèmes qui lui sont particulièrement chers, le « désir d'écrire » pointe sous la plume de Hardy : le monde du livre ; la question religieuse - le Paris janséniste, le devenir des exjésuites, la santé de l'archevêque de Paris, Christophe de Beaumont, sa bête noire - ; certains procès devenus des affaires publiques mettant en cause des membres de l'aristocratie ; les réformes du chancelier Maupeou et la vague de Maupeouana pamphlets hostiles - qu'elles suscitent. Hardy baisse alors brièvement la garde et se livre un peu, souvent sous la forme de confidences morales. Comme l'écrit Pascal Bastien dans la présentation du premier volume de l'édition de Mes Loisirs :

On peut imaginer Hardy se promener dans les rues de Paris, noter ses observations dans un carnet, multiplier chez lui ses aide-mémoire et réunir, dans son cabinet de travail, les écrits qui n'étaient pas de sa plume. Après quelques jours peut-être, le temps de vérifier ou compléter l'information, il rédigeait une première version de son Journal, consultant ses notes et les augmentant de ses propres souvenirs ou de récentes nouvelles qu'il avait pu recueillir. Enfin il recopiait ses Loisirs dans ses grands registres, attentivement, scrupuleusement, avec des renvois, des compléments d'information ou des remarques personnelles ; la fatigue et l'inattention inhérente au travail de copiste se remarquent, d'ailleurs, à travers une ligne ou un titre mal retranscrit. Mes Loisirs est une vocation. ${ }^{11}$

\footnotetext{
${ }^{10}$ Journal de ma vie. Jacques-Louis Ménétra, compagnon vitrier au XVIIIe siècle, présenté par Daniel Roche, Paris, éd. Montalba, 1982, rééd. Albin Michel, 1998, présentation.

${ }^{11}$ Pascal Bastien, «Présentation », Siméon-Prosper Hardy, Mes Loisirs ou Journal d'événements tels qu'ils parviennent à ma connoissance, éd. par Pascal Bastien et Daniel Roche, Paris, Hermann, 2012, volume 1 (1753-1770), p. 18.
} 
Même si son Journal d'événements ne livre pas d'impressions de lecture personnelles, Hardy arpente un territoire, celui du Paris janséniste, et tout particulièrement les paroisses Saint-Séverin et Saint-Étienne-du-Mont, où il s'est trouvé domicilié à un moment ou un autre des vingt-cinq années de son journal. Inséré dans un dense réseau de relations amicales, il tire de nombreuses informations de ses «loisirs », de ses lectures et des conversations avec ses fréquentations ecclésiastiques, gens de justice et gens du livre notamment. Il les complète par des libelles et des pamphlets, ces «imprimés furtifs » qu'il reçoit par toutes sortes de canaux, comme lors des promenades qu'il fait dans les jardins du Palais-Royal, du Luxembourg ou des Tuileries, où se retrouvent les nouvellistes et les mouches de police. À la mort de ce vieux libertin qu'était le maréchal duc de Richelieu, il note par exemple : «Je ne peux malheureusement comme historien me dispenser de placer ici deux pièces satyriques $($ sic $)$ autant qu'injurieuses à sa mémoire $»^{12}$. Hardy livre ainsi une histoire au présent, au quotidien même, avec ses soubresauts, ses accalmies, mais aussi avec le recul de la mise en forme et au propre. Le geste - «je ne peux [...] me dispenser » - mérite d'être considéré, car Hardy l'identifie sans équivoque à celui de l'historien. Il mérite donc mieux que ce qu'en dit Jules Flammermont : «En prenant cette peine le bonhomme croit faire œuvre d'historien $»^{13}$

Hardy est également amené, en raison de ses fonctions de syndic adjoint de la communauté des libraires pendant le «coup Maupeou », à traiter avec la direction de la Librairie et avec Sartine, alors lieutenant général de police de Paris. C'est notamment le cas lorsqu'un exemplaire d'une nouvelle édition du célèbre livre pornographique Dom B[ougre], portier des Chartreux, attribué à Jean Gervaise de La

\footnotetext{
${ }^{12}$ Mes Loisirs, 11 août 1788.

13 Jules Flammermont, La journée du 14 juillet 1789. Fragment des mémoires inédits de L. G. Pitra, électeur de Paris en 1789, Paris, Société de l'Histoire de la Révolution française, 1892, «Introduction », p. CXLVI, note 2.
} 
Touche, est intercepté par les douaniers, en provenance de Calais. Significativement, Hardy apprécie d'abord l'ouvrage en homme de l'art : «très jolie édition avec figures en taille douce, sous la date de 1771 , in $-8^{\circ} »$ et, en le lisant, on l'imagine clairement en train de manipuler le livre avec l'œil expert du métier. L'épître dédicatoire scandaleuse qu'il y découvre alors, l'amène à se rendre avec ses collègues auprès de Sartine. Mais après coup, Hardy confie à son journal ses interrogations et celles de ses confrères, tout en se demandant si elles sont fondées... Le lieutenant-général de police n'a-t-il pas voulu tester leur fidélité, alors que le nombre des libelles et des pamphlets explose au début des années 1770 ?

En combinant après coup, les différentes circonstances de cet événement singulier, comme nous n'ignorions pas que le corps de la librairie et imprimerie, avait un grand nombre d'ennemis, il nous vint dans l'esprit que ce pouvait bien être une espèce de piège qui nous avait été tendu, pour mettre à l'épreuve notre vigilance et notre exactitude, qui pourtant n'auraient pas dû être suspectées tant nous prenions de précautions pour ne point donner prise sur nous ; au surplus il était très possible qu'il y eût plus de terreur panique que de réalité, dans nos conjectures à cet égard. ${ }^{14}$

On a là un exemple concret de notations personnelles qui relèvent à la fois de l'histoire matérielle du livre, de son histoire institutionnelle - les rapports du monde du livre avec la Librairie-, et de l'histoire politique immédiate.

\section{III. À l'heure des humanités numériques}

Il se trouve qu'au sein du projet de recherches CITERE consacré aux «Circulations Territoires et Réseaux en Europe de l'âge classique au siècle des Lumières » que j'ai dirigé dans le cadre de l'Agence nationale de la Recherche à

\footnotetext{
${ }^{14}$ Mes Loisirs, 8 février 1768.
} 
partir de $2008,{ }^{15}$ plusieurs d'entre nous ont travaillé sur le monde du livre au XVIII ${ }^{\mathrm{e}}$ siècle, notamment à partir de l'analyse spatiale de l'implantation des bibliothèques dans la ville et de leurs relations avec leurs fournisseurs, ${ }^{16}$ ou encore d'archives policières sur le livre interdit. Daniel Droixhe a ainsi étudié un réseau de colporteuses de livres clandestins à Paris, actif en 1766, puis démantelé par la police, qui cherche lors des interrogatoires non seulement à en préciser les ramifications et à identifier les complices, mais aussi à les situer dans l'espace parisien. ${ }^{17}$ La synthèse cartographique réalisée à l'occasion de cette étude à partir de l'ensemble des données recueillies montre qu'il est tout à fait possible de proposer dans le même esprit, non seulement une carte du monde du livre chez Hardy, mais aussi une carte de synthèse des lieux où il se procure ses informations, recueille pamphlets et nouvelles, ainsi que des personnes-sources qui les lui communiquent -quand elles sont mentionnées. On peut appliquer le même traitement à un autre versant passionnant des Loisirs, celui du Paris janséniste de Hardy, en variant les échelles d'observation depuis sa paroisse d'appartenance. C'est pourquoi il faut se réjouir de la toute récente création au sein du département d'histoire de l'Université du Québec à Montréal du Laboratoire Paris XVIII qui se propose, sous la direction de Pascal Bastien, de « reconstituer la mobilité et les usages de l'information dans le Paris des Lumières, parce que cette information est le principal matériau d'une archéologie de la citoyenneté prérévolutionnaire $»^{18}$.

\footnotetext{
${ }^{15} \mathrm{http}: / /$ citere.hypotheses.org/ consulté le 20 décembre 2017.

16 Emmanuelle Chapron, Anne Saada, «Les bibliothèques, la carte et le territoire », Pierre-Yves Beaurepaire (dir.), La communication en Europe, de l'âge classique au siècle des Lumières, Paris, Belin, 2014, p. 215-264.

${ }^{17}$ Daniel Droixhe, «Un essai de topographie et d'économie du livre prohibé. La rafle des colporteuses parisiennes de 1766 », Pierre-Yves Beaurepaire (dir.), La communication en Europe, de l'âge classique au siècle des Lumières, op. cit., p. 265-292.

18 Citation extraite de la présentation du Laboratoire Paris XVIII : http://www.grhs.uqam.ca/laboratoire-paris-xviii/ consulté le 20 décembre 2017.
} 
L'instrumentation électronique développée dans les recherches sur les egodocuments, notamment lors du projet «Le Monde de Corberon » il y a une décennie, peut elle aussi être appliquée à Mes Loisirs. Dans ce projet expérimental relevant des humanités numériques, Dominique Taurisson et moi avions pour but de reconstituer, à partir de son journal, l'environnement personnel, amical, professionnel, mais aussi culturel et spatial d'un diplomate de rang moyen, Marie-Daniel Bourrée, chevalier puis comte de Corberon, avant et après son départ en Russie avec l'ambassade du marquis de Juigné. ${ }^{19}$ Ce journal tenu sous la forme de lettres fictives avait été publié au début du $\mathrm{XX}^{\mathrm{e}}$ siècle par Léon-Honoré Labande mais avec de nombreuses coupes. ${ }^{20}$ La source est intéressante non seulement par la précision de ses notations, mais aussi par l'intense vie sociable du diariste, et son attention à recueillir un maximum d'informations pour préparer sa mission diplomatique. Dans le système Arcane, du nom de l'instrumentation électronique développée par Eric-Olivier Lochard, que nous avions utilisée, chaque nom, chaque lieu, chaque institution, chaque événement mentionné dans le journal est un sujet de la base de données relationnelles. ${ }^{21}$ Tout un éventail de relations créées par les chercheurs eux-mêmes en fonction de leurs centres d'intérêt, et accessibles en menu déroulant, sont indexées sur la transcription intégrale du texte mais peuvent aussi être reportées directement sur les numérisations correspondantes de la source. Le lecteur peut ensuite interroger la base de données pour afficher les occurrences d'une relation -par exemple «parler de » Voltaire ;

\footnotetext{
${ }^{19}$ Parmi différentes publications : Pierre-Yves Beaurepaire, «L’instrumentation électronique appliquée aux écrits du for privé. Le projet «Le monde de Marie-Daniel Bourrée de Corberon », Jean-Pierre Bardet, François-Joseph Ruggiu éd., Au plus près du secret des cœurs ? Nouvelles lectures historiques des écrits du for privé en Europe du XVIe au XVIIIe siècle, Paris, Presses de l'Université de ParisSorbonne, 2005, p. 247-253.

${ }^{20}$ Léon Honoré Labande, Un diplomate français à la cour de Catherine II (1775-1780), journal intime du chevalier de Corberon, Paris, Plon-Nourrit, 1901, 2 vol.

${ }_{21}$ Eric-Olivier Lochard et Dominique Taurisson, «'Le monde selon Arcane' : un paradigme instrumental pour l'édition électronique », Cahiers GUTenberg, n³9-40, mai 2001, p. 89-105.
} 
« recommander » une pièce de théâtre- ou les croiser «parler de franc-maçonnerie et de Russie $»^{22}$.

Si le texte de Mes Loisirs est moins personnel que celui du journal de Corberon, on a vu plus haut que des traces de réaction plus personnelle à des événements, à des rencontres ou à des nouvelles existent cependant. Comme dans les deux cas la transcription intégrale de la source existe, un outil d'instrumentation électronique, du type d'Arcane, permet de travailler avec efficacité et précision sur «Le Monde de Hardy» en référençant lieux, personnes, institutions, objets, mais surtout en indexant des relations comme parler des billets de confession, de l'archevêque de Paris, du prix des grains, enregistrer les morts violentes portées à sa connaissance, etc., parmi beaucoup d'exemples et de formulations possibles des dites relations. Il sera ensuite très aisé d'interroger systématiquement la source ou de «tester » des croisements de relations pour voir si les interrogations sont pertinentes. Autant de possibilités qui existent sans instrumentation électronique, mais qui sont extrêmement chronophages dans le cas d'un corpus étendu, ce qui a souvent un caractère dissuasif. C'est ce que propose désormais à la communauté scientifique le Laboratoire Paris XVIII à travers sa plate-forme logicielle POLIS qui associe un système d'information géographique à une base de données regroupant « un corpus d'archives diversifiées, jusqu'ici dispersées et rarement mises en relation, rendant enfin possible l'accrétion, le croisement et l'interrogation de documents du XVIIIe siècle inédits et manuscrits, imprimés et publics, qui se sont toujours ignorés et que l'infrastructure peut désormais identifier dans l'espace urbain et suivre, d'une manière dynamique et croisée, sur une carte de l'époque $»^{23}$. Parmi ces premiers gisements mis

\footnotetext{
${ }^{22}$ Des captures d'écran des résultats graphiques de ces requêtes sont disponibles dans les différents articles sus-mentionnés.

${ }^{23}$ http://www.grhs.uqam.ca/laboratoire-paris-xviii/ consulté le 20 décembre 2017.
} 
en exploitation figure justement le journal d'événements de Siméon-Prosper Hardy, aux côtés d'autres types de supports complémentaires d'information: Gazette de France, nouvelles à la main et gazetins de police. Il devient dès lors possible de « reconstituer, au ras du sol, la confrontation des forces, l'émergence de nouvelles idées et l'apparition de nouvelles identités. La population, les conflits, le langage, les échanges et le commerce qui se réunissent dans un quartier, un voisinage, un carrefour ou une rue annoncent chez les acteurs un engagement personnel vers des enjeux publics, qui entend se réaliser à travers d'autres formes de solidarité et des structures insoupçonnées de sociabilité ${ }^{24}$.

Grâce à ces nouveaux outils, la rumeur comme vecteur d'information et de désinformation, comme catalyseur ou réactif, peut ainsi être étudiée à nouveaux frais, en la restituant dans sa dynamique spatiale- ce qui avait été tenté dans le cadre du projet CITERE à propos de la diffusion d'une rumeur politique dans l'espace urbain madrilène au XVII ${ }^{\mathrm{e}}$ siècle, lorsque Don Juan d'Autriche marche sur la ville avec ses partisans. ${ }^{25}$ Parmi les nombreuses rumeurs dont Mes Loisirs se fait l'écho, citons cette notation du 7 février 1773 : «Ce jour on apprend que l'Université était encore toute en rumeur ». Hardy note dans son journal les vers hostiles à Maupeou qu'il glane en société ou au café. Mais le libraire, s'il n'est pas favorable à la politique du chancelier, a soin de prendre ses distances, car c'est un homme d'ordre, respectueux de l'autorité et des conventions : «Un autre particulier plus téméraire encore et bien plus répréhensible avait eu la hardiesse de composer contre lui et contre le chancelier, les vers suivants que je transcris ici uniquement comme faisant partie de l'histoire du temps ; mais sans aucune approbation des injures atroces qu'ils contiennent » (8

\footnotetext{
${ }^{24} \mathrm{http}: / /$ www.grhs.uqam.ca/laboratoire-paris-Xviii/ consulté le 20 décembre 2017.

${ }^{25}$ Héloïse Hermant, «Les dispositifs de communication de don Juan José de Austria et l'orchestration d'un mouvement d'opinion », Pierre-Yves Beaurepaire (dir.), La communication en Europe de l'âge classique aux Lumières, op. cit., p. 162-174.
} 
février 1770). On aura à nouveau remarqué la référence à l'histoire, dès que la notation, ou plutôt le choix de recueillir tel écho, se fait plus personnel :

Maupeou que Dieu dans sa colère Nomma l'organe de nos lois ;

Beaucoup plus fourbe que son père Et plus scélérat mille fois

À, pour combler notre misère

De l'odieux Terray fait choix.

Le lâche ! Il lui faut un complice,

Mais il trouvera son supplice

Dans le cour de l'abbé sournois. ${ }^{26}$

\section{En guise d'ouverture : de l'écriture de la solitude chez Corberon au lexique de l'indignation chez Hardy}

L'instrumentation électronique appliquée au journal de Corberon avait permis par exemple d'indexer l'ensemble des références explicites ou implicites à la solitude que le scripteur choisit de confier à son ego-document. ${ }^{27}$ De la même manière, ou même en traitant la source avec un outil logométrique comme Hyperbase, ${ }^{28}$ il devient possible d'étudier ce que l'on pourrait nommer «le lexique de l'indignation » chez Hardy. En effet, la lecture de Mes Loisirs montre que les affaires judiciaires qui opposent des représentants de l'aristocratie à des gens de peu l'émeuvent profondément. Le libraire insiste sur les négociations et manœuvres entreprises pour

\footnotetext{
${ }^{26}$ Mes Loisirs, 8 février 1770.

27 Dominique Taurisson, «Solitude et espaces relationnels du chevalier de Corberon Paris-SaintPétersbourg-Paris (1775-1781), Jean-Pierre Bardet, Élisabeth Arnoul et François-Joseph Ruggiu (dir.), Les écrits du for privé en Europe (du Moyen Âge à l'époque contemporaine). Enquêtes, Analyses, Publications, Talence, Presses universitaires de Bordeaux, 2009, p. 247-266.

${ }^{28}$ Voir notamment Véronique Magri-Mourgues, « Analyse textométrique et interprétation littéraire Hyperbase, Rousseau et les Lumières », TRANEL. Travaux Neuchâtelois de Linguistique, Institut des sciences du langage et de la communication (Neuchâtel, Suisse), 2012, La linguistique de corpus - de l'analyse quantitative à l'interprétation qualitative, p.77-93 et par extension l'ensemble des recherches développées à l'Université Côte d'Azur au sein du laboratoire Bases, Corpus, Langage UMR CNRS 7320 autour de «Logométrie et corpus politiques, médiatiques et littéraires »: http://logometrie.unice.fr/ consulté le 20 décembre 2017.
} 
étouffer la rumeur et tordre les bruits qui parcourent la ville, et ne cache pas sa réprobation morale devant l'impunité dont certains bénéficient. Il relate ainsi l'affaire

à laquelle le duc de Fronsac, fils du maréchal duc de Richelieu, est directement mêlé en 1768 et qui aurait eu sa place dans le livre de Sarah Maza, Vies privées, affaires publiques. Les causes célèbres de la France prérévolutionnaire. ${ }^{29}$

Du lundi premier février [1768]

Il se répand dans le public que M. le duc de Fronsac, fils du maréchal duc de Richelieu, veuf depuis quelque temps, qui avait conçu la passion la plus vive pour une jeune demoiselle très jolie fille d'un ancien marchand de la rue SaintHonoré qu'il suivait depuis fort longtemps, et qui se trouvait fêtes et dimanches à la même messe que la mère et la fille avaient coutume d'entendre dans l'église des religieuses de la Magdeleine de la Ville L'Evêque, au sortir de laquelle église, il prit une fois sur lui de les accoster toutes deux, et de faire à la mère différentes propositions se répandant beaucoup en éloges sur la beauté de la fille ; propositions que la mère avait constamment rejetées, et qui lui firent même prendre le parti d'aller entendre la messe dans d'autres églises ; ce qui n'avait pas empêché que le duc de Fronsac ne s'obstinât toujours à la suivre, comme auparavant; jusqu'à ce qu'enfin las de faire de vaines poursuites, il se détermina à user d'un stratagème assez singulier qui fut d'aller un soir assez tard avec un nombre de gens apostés crier au feu près du logis de cette dame, cherchant à lui persuader que le feu était chez elle, pour l'engager à sortir de son appartement avec sa fille, faisant même plus et les déterminant à descendre dans la rue pour observer où était le feu, ce qui réussit assez bien pour que la demoiselle s'étant trouvée un instant sans madame sa mère, on saisit l'occasion pour lui jeter une couverture sur la tête, l'enlever et la placer dans un carrosse qui n'était pas loin de là ; la mère revenue de son effroi, et cherchant en vain sa fille qu'elle avait crue remontée chez elle, après avoir fait inutilement toute perquisition possible dans le voisinage, fut porter ses plaintes à $\mathrm{M}$. le lieutenant de police, qui mit sur le champ son monde en campagne pour découvrir ce que la jeune personne pouvait être devenue, on la trouva suivant les uns dans les vingt-quatre heures, et suivant d'autres au bout de trois jours chez une femme de mauvaise vie, elle donna les preuves de la plus vive douleur à l'exempt qui fit cette découverte, annonçant qu'elle était une fille perdue et déshonorée ; M. le lieutenant de police ayant fait en Cour son rapport sur cette affaire ; les ducs et pairs s'assemblèrent plusieurs fois pour aviser au parti qu'il y aurait à prendre sur un délit aussi grave commis par un d'entre eux ; pendant leurs assemblées et avant qu'ils eussent statué, des personnes de considération, s'employèrent pour négocier un accommodement, et persuadèrent aux père et mère de la demoiselle qui d'abord paraissaient bien décidés à poursuivre la réparation d'un outrage aussi sanglant partout où il serait convenable de le faire, de renoncer à toute poursuite, et de recevoir comme par forme de dommages et intérêts, une somme de cent mille livres suivant quelques personnes, d'autres ont dit, douze mille livres de pension viagère et vingt quatre mille livres d'argent comptant $[\ldots]^{30}$

\footnotetext{
${ }^{29}$ Sarah Maza, Vies privées, affaires publiques. Les causes célèbres de la France prérévolutionnaire, traduit de l'anglais par C. Beslon et P.-E. Dauzat, Paris, Fayard, 1997.

${ }^{30}$ Mes Loisirs, $1{ }^{\text {er }}$ février 1768.
} 
Malgré l'utilisation du pronom impersonnel, le commentaire de Hardy est bien présent :

On chercha ensuite à étouffer l'affaire entièrement en semant dans le public que le fait était faux; on ne laissa pas néanmoins que de le tenir pour certain, et de gémir en secret de voir demeurer impuni dans la personne d'un Grand, un crime horrible qui eut fait éprouver les châtiments les plus rigoureux, à tout autre particulier qui eut eu le malheur de s'en rendre coupable. ${ }^{31}$

Sous sa plume, la relation de ces affaires n'est donc pas neutre, mais terminée par un jugement moral, témoignage du désarroi que les errements de la justice peuvent créer. À propos d'une affaire de sévices sexuels infligés à une jeune femme par le comte de Sade, qu'il relate le 8 avril 1768, Hardy conclut ainsi :

Quoi qu'il en soit ce trait aussi singulier qu'il est infâme et révoltant, si la justice n'en prend connaissance, et n'en fait un châtiment exemplaire, fournira à la postérité un exemple de plus de l'impunité qui suit d'ordinaire dans notre siècle les crimes les plus abominables, dès que ceux qui les commettent ont le bonheur d'être grands, riches ou accrédités. ${ }^{32}$

Faites à la lumière de projets de recherche en humanités numériques, qui associent édition de sources et développement de nouveaux outils d'analyse pour l'historien, ces perspectives montrent la richesse et le potentiel remarquables des Loisirs. Elles s'appuient toutes sur les manifestations du désir d'écrire qui perlent d'un journal d'événements et qui permettent de brosser par petites touches le portrait moral de son scripteur.

\footnotetext{
${ }^{31}$ Ibid.

${ }^{32}$ Mes Loisirs, 8 avril 1768.
} 